\title{
STUDY OF CLINICAL OUTCOME OF TENZEL FLAP IN UPPER EYELID RECONSTRUCTION
}

\author{
Manjula Y. $M^{1}$
}

${ }_{1}^{1}$ Assistant Professor, Department of Ophthalmology, BGS Global Institute of Medical Sciences.

\begin{abstract}
BACKGROUND

Resection of upper eyelid tumour, benign or malignant, is one of the most common cause of upper eyelid defect, followed by trauma. Considering the normal eyelid structure, reconstruction of anterior and posterior lamella of eyelid for proper functional and cosmetic results is quite a challenging work. Upper eyelid reconstruction is done by various methods depending on age of the patient, location of defect and amount of eyelid defect.

Aim-To study the clinical outcome of Tenzel flap in upper eyelid reconstruction in cases with 30-50\% of Full-thickness Upper eyelid defect.
\end{abstract}

\section{MATERIALS AND METHODS}

This study was conducted from April 2013-April 2016, in BGS GIMS Medical College and Hospital that included 12 patients with $30-50 \%$ of Full-thickness Upper eyelid defect which were reconstructed by Tenzel flap. They were followed up for one year postoperatively.

\section{RESULTS}

In our study, out of 12 patients, the causes for 30-50\% Full-thickness Upper eyelid defect was due to excision of benign meibomian gland tumour in 7 patients and ocular trauma in 5 patients. All patients underwent Tenzel flap upper eyelid reconstruction without any intraoperative or postoperative complication. During followup period, no recurrence of tumour, lagophthalmos, hypertrophic scar or abnormally thick lid appearance was noted. Results were good in all patients cosmetically and functionally after one year of followup.

\section{CONCLUSION}

Tenzel flap in upper eyelid reconstruction is a good technique for $30-50 \%$ of Full-thickness Upper eyelid defect with no complication and acceptable cosmetic and functional outcome.

\section{KEYWORDS}

30-50\% Full-thickness Upper Eyelid Defects, Tenzel Flap, Upper Eyelid Reconstruction, Meibomian Gland Tumour, Ocular Trauma. HOW TO CITE THIS ARTICLE: Manjula YM. Study of clinical outcome of Tenzel flap in upper eyelid reconstruction. J. Evolution Med. Dent. Sci. 2018;7(03):269-272, DOI: 10.14260/jemds/2018/60

\section{BACKGROUND}

Eyelids are protective curtains of an eye ball. Eyelid reconstruction applies to defects resulting from tumour resection as well as congenital and traumatic defects. The incidence of ocular injuries in craniofacial trauma is high ranging from $15-60 \%$,(1) several methods may be appropriate for reconstruction of particular eyelid defect. The choice of procedure depends on age of the patient, the condition of the eyelids, specific adnexal structures involved,(2) the size and position of the defect.

\section{Priorities in Eyelid Reconstruction are-}

1. Adequate eyelid closure.

2. Development of stable eyelid margin.

3. Provision of adequate vertical eyelid height.

4. Smooth, epithelialised internal surface.

5. Maximum cosmesis and symmetry.

'Financial or Other Competing Interest': None.

Submission 07-11-2017, Peer Review 28-12-2017,

Acceptance 03-01-2018, Published 13-01-2018.

Corresponding Author:

Manjula Y. $M$

Assistant Professor,

Department of Ophthalmology,

BGS Global Institute of Medical Sciences.

E-mail: manjumahesh50@gmail.com

DOI: $10.14260 /$ jemds $/ 2018 / 60$

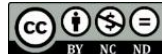

Eyelid injuries that result in tissue loss provide a difficult reconstructive challenge. If a full-thickness loss of eyelid tissue leads to lagophthalmos and corneal exposure, aggressive lubrication with antibiotic ointments is instituted or a temporary tarsorrhaphy placed until definitive repair can be accomplished.

Tissue loss that involves only the anterior skin muscle lamella may be repaired, if the tissue is small and defect lies anterior to the orbital septum by allowing the defect to granulate. This method may obviate the need for skin grafts and myocutaneous flaps, however, the wound must be monitored carefully for infection and late contracture. The result of allowing the eyelid to granulate spontaneously can be equal to, or surpass, the outcome of primary repair.

Full-thickness eyelid lacerations that do not involve the eyelid margin may be associated with significant internal disarrangement of lid structures and perforation of the globe. The injuries require adequate layer by layer inspection of the wound to assess the integrity of the orbital septum, levator muscle and levator aponeurosis, conjunctiva, rectus muscle, and the globe. Meticulous layered closure is required, with the septum left unsutured.

If the posterior lamella of the eyelid is involved in a fullthickness eyelid injury but can be re-approximated without undue tension, it is repaired directly. Tarsal alignment is achieved best through interrupted buried sutures. In the upper eyelid, these sutures must be passed through the 
tarsus but remain subconjunctival because full thickness sutures may result in corneal contact and irritation.

When an injury is severe enough to result in full thickness tissue loss that involves both the anterior and posterior lamellae of the eyelid, the technique of repair depends on the amount and location of the tissue loss. Many of the techniques for such injuries are used in lid reconstructions implanted after eyelid skin cancer resections.

The amount of tissue loses can usually be ascertained only after careful re-approximation of wound. Fortunately, tissue loss is usually less significant than the initial presentation may suggest, as retraction of the tissues gives the appearance of greater tissue loss than actually has occurred.

Every effort is made to preserve all tissue. The generous adnexal vascular supply usually preserves even narrow pedicles and even largely avulsed tissue can be reattached with significant survival rates. If large defect persists, standard methods of eyelid reconstruction are employed to complete the anatomic repair.

\section{The following Principles Guide the Practice of Eyelid Reconstruction-}

- Reconstruction of either anterior or posterior eyelid lamella not both.

- Maximise horizontal tension and minimise vertical tension.

- Maintain sufficient and anatomical canthal fixation.

- Narrow the defect as much as possible before sizing a graft.

Small superficial eyelid lacerations not involving lid margin parallel to skin tension lines are stabilised by skin tape. Lacerations that are perpendicular to the skin tension lines require careful approximation and eversion of skin edges by 6-0 non-absorbable sutures. Adnexal trauma needs most meticulous eyelid approximation to avoid eyelid notching and margin malposition.(3)

Upper eyelid full-thickness eyelid defect with $<30 \%$ of horizontal tissue loss is repaired by primary closure after smoothing the wound margin by 6-0 non-absorbable sutures placed at meibomian glands opening at lid margin $2 \mathrm{~mm}$ from wound edge and $2 \mathrm{~mm}$ deep, tarsus are closed with interrupted partial thickness 6-0 Vicryl or 7-0 silk. $(4,5)$ knots are tied anterior to tarsal surface to avoid corneal irritation, skin is closed by 6-0 silk.

Upper eyelid full-thickness eyelid defect with $30-60 \%$ of horizontal tissue loss is reconstructed by primary closure as described above after release and advancement of lateral tissue by lateral canthotomy followed by cantholysis.

Tenzel flap is a semi-circular skin and muscle rotational flap used for upper eyelid reconstruction(6,7,8) of defect created by excision of benign big meibomian gland tumour and trauma in our study.

Flap starts from lateral canthus directed semi-circular incision downwards for upper lid defect, canthotomy and cantholysis done, skin and muscle flap rotated to defect and closed primarily. Lateral canthal tendon reconstructed using silk suture.

The advantage of this procedure is that it is a one stage complete procedure. Disadvantage is excessive long scar on face.
Upper eyelid full-thickness eyelid defect $>60 \%$ is constructed in two stages; lid sharing procedure like Modified Hughes procedure performed in 2 stages, eye is closed for several days after the operation as flap is preserved attached to its origin for several days. $(9,10)$ The technique encompasses a tarsoconjunctival advancement flap from upper lid for the posterior lamella and skin graft or linear advancement flap for anterior lamella. Lower lid wound is modified to a rectangular defect, same measurement is transferred to undersurface of everted upper lid. The rectangular tarsoconjunctival flap is dissected from levator aponeurosis dissecting upper forniceal attachment.

The tarsoconjunctival flap is placed in lower lid defect and sutured with approximation of tarsus to tarsus. For anterior lamella, free skin graft is used, taken from retroauricular sulcus, supraclavicular area and sutured using 6-0 silk. Release of the Hughes procedure should be accomplished only when the reconstructed lid is as supple as the opposite lower lid. Minimum timeframe for this is 6-8 weeks to months based on patient healing potential. Once the flap is separated from the surface of the globe, it is severed as close to its upper lid origin and any excess above the lower lid margin is excised.

Cutler-beard bridge flap is used to repair large defect of upper lid. Similar to Hughes flap, first skin muscle and conjunctiva are advanced from lower eyelid, $2^{\text {nd }}$ stage of excision done after 3-4 weeks. A significant problem with this procedure is lack of rigid structure in the reconstructed lid, so autogenous cartilage is used to augment the posterior lamella.

\section{MATERIALS AND METHODS}

In this study, 12 patients who visited BGS GIMS Ophthalmology Department during the period April 2014 to April 2016 who had upper eyelid defect $30-60 \%$ due to trauma in 5 patients. Excision of moderately big adnexal tumour of upper lid was in 7 patients, they underwent Tenzel flap upper lid reconstruction. All were analysed and followed for one year postoperatively.

Ethical committee clearance from the institution was obtained.

Detailed history of trauma was obtained in 5 cases with traumatic upper eyelid defect. Ocular examination was done using slit lamp. In all patients, visual acuity was $6 / 6$ in both eyes with no open globe injury. Cases with open globe injury was excluded in our study.

Seven patients presented with medium-sized meibomian gland tumour, detailed history of duration of onset of progression was taken. Ocular examination done by slit lamp, in all cases it was normal. They were posted for excision and biopsy with upper eyelid reconstruction by Tenzel flap. In all cases, histopathology showed benign or inflammatory meibomian gland tumours.

Complete tetanus immunisation is done depending on the status of patient immunisation.(11)

Informed consent was taken in all patients.

12 patients with $30-60 \%$ of Full-thickness Upper eyelid defect underwent Tenzel flap reconstruction under general anaesthesia. The margin of eyelid defect was smoothed, lateral canthotomy incision was made, semi-circular incision made i.e. inferiorly arching line for upper eyelid defect, the arc had a diameter of about $2 \mathrm{~cm}$. The canthotomy was made 
followed by severing of the lateral canthal ramus of the mobilised lid. Ramus of opposite lid was preserved, undermining of temporal tissue was done to mobilise the adjacent tissues. Repair was done by lid margin suturing by 6-0 silk, tarsoconjunctival alignment was done using 7-0 Vicryl suture, subcutaneous tissue and skin was closed by 6-0 silk. Moxifloxacin eye ointment applied, pad and bandage done.

$1^{\text {st }}$ postoperative day dressing was done, topical antibiotic eye ointment continued 3 times a day.

Ice compression was given for first two days. Wound was left open, no bandage was done.

Systemic tablet ciprofloxacin $500 \mathrm{mg}$ twice daily was given for 5 days.

Tablet Combiflam was given twice a day for three days. Skin suture was removed on 7 th postoperative day, lid margin suture was removed in the second week $\left(10^{\text {th }}-14^{\text {th }}\right.$ postoperative day).

All patients were followed up at 1 week, 1 month, 3 months, 6 months and one year.

\section{RESULTS}

In our study, 12 patients with 30-60\% Full-thickness Upper eyelid defect underwent Tenzel flap lid upper eyelid reconstruction-

- $\quad$ Age varied from 20 to 60 years.

- Male to female ratio was 8:4.

- Causes for eyelid defect was ocular trauma in 5 cases, all were male patients, age was between 20 to 30 years.

7 cases were due to upper lid meibomian gland tumour excision. Histopathology confirmed all as benign meibomian gland tumours. There was no complication during surgery and after surgery. All patients were followed postoperatively $1^{\text {st }}$ day, 1 week, 1 month, 3 months, 6 months and 1 year.

Skin suture was removed after $7^{\text {th }}$ postoperative day. Lid margin suture was removed after $10-15^{\text {th }}$ postoperative day. All patients' results were satisfactory aesthetically and functionally. None of the patients had ectropion, lid retraction, lid notching, lid movement was normal in all patients at the end of one year of followup.
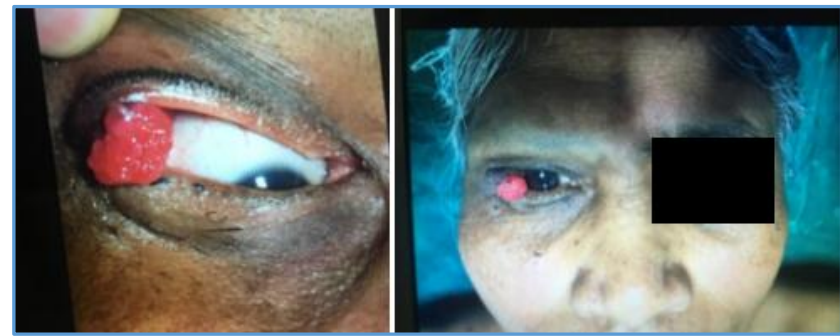

Figure 1. Meibomian Gland Tumour of Right Upper Lid

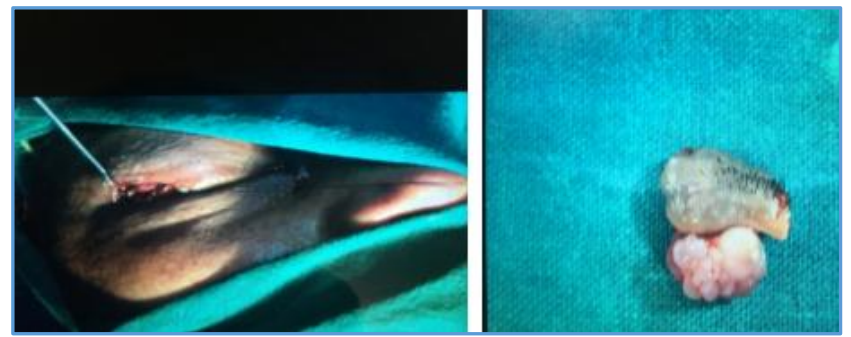

Figure 2. Excision of Meibomian Gland Tumour Creating Full-thickness Upper Eye Lid Defect

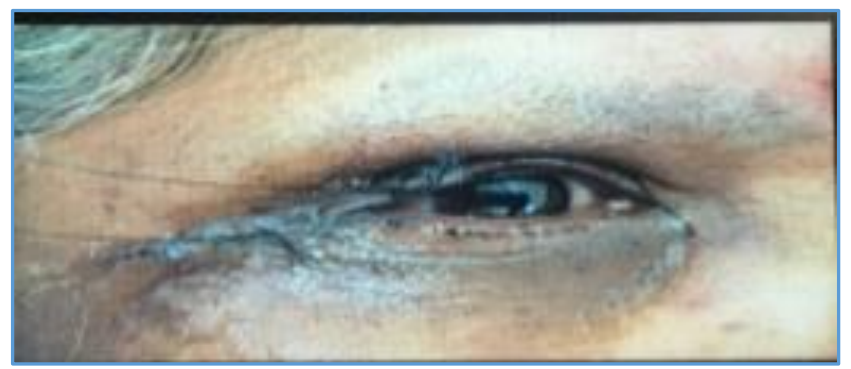

Figure 3. Reconstruction of Upper Eye Lid Defect by Tenzel Flap

\section{DISCUSSION}

Upper eyelid reconstruction after full-thickness eyelid defect due to trauma, excision of eyelid tumour or congenital defect is quite challenging.

To provide optimal, anatomical, aesthetic and functional results of reconstruction of the eyelids, several methods have been introduced considering age of the patient, location of the lesion, size of defect, $(7,8)$ specific adnexal structure involved.

In our study, 12 patients with $30-60 \%$ of upper eyelid full-thickness defect were reconstructed by Tenzel flap in a single session, which showed good cosmetic and functional result. There was no postoperative complication except semicircular incision mark. Longterm followup is necessary to know about longterm results as some of the complication might appear in the longer course of the time. But there was no significant complication in our study. None of our case had ectropion, lagophthalmos or eyelid margin abnormality.

\section{CONCLUSION}

Resection of upper eyelid tumour benign or malignant is one of the most common cause of upper eyelid defect, followed by trauma. Considering the normal eyelid structure, reconstruction of anterior and posterior lamella of eyelid for proper functional and cosmetic results is quite a challenging work.

Upper eyelid reconstruction is done by various methods depending on age of the patient, location of defect, amount of eyelid defect. Tenzel flap upper eyelid reconstruction in 30$50 \%$ upper eyelid full-thickness defect is a simple and safe, one-staged technique with no-to-minimal complication except a semi-circular incision mark on face. However, longterm followup is necessary to know about longterm results.

\section{REFERENCES}

[1] Bowman PH, Fosko SW, Hartstein ME. Periocular reconstruction. Semin Cutan Med Surg 2003;22(4):263-72.

[2] Codner MA, McCord CD, Mejia JD, et al. Upper and lower eyelid reconstruction. Plastic Reconstruct Surg 2010;126(5):231e-45e.

[3] Murchison AP, Bilyk JR. Management of eyelid injuries. Facial Plast Surg 2010;26(6):464-81.

[4] Price DL, Sherris DA, Bartley GB, et al. Forehead flap periorbital reconstruction. Arch Facial Plastic Surg 2004;6(4):222-7.

[5] Verity DH, Collin TR. Eyelid reconstruction: the state of art. Curr Opin Otolarynsol Head Neck Surg 2004;12(4):344-8 
[6] Garces JR, Guedes A, Alegre M, et al. Double mucosal and myocutaneous island flap: a one - stage reconstruction for full-thickness lower eyelid defect. Dermatol Surg 2009;35(5):779-85.

[7] Gunduz K, Demirel S, Gunalp I, et al. Surgical approaches used in reconstruction of the eyelids after excision of malignant tumors. Ann Ophthalmol (Skokie) 2006;38(3):207-12.

[8] Steinkogler FJ. Reconstruction of the lower lid. Br J Ophthalmol 1984;68(7):507-10.
[9] Jacob V, Mokal NJ, Deshpandae SN. Bi-lamellar lower eyelid reconstruction with superficial temporal artery island flap and hard palate muco-periosteal free graft. Indian J Plast Surg 2005;38(2):105-9.

[10] Marks MW, Argenta LC, Friedman RJ, et al. Conchal cartilage and composite grafts for correction of lower lid retraction. Plast Reconst Surg 1989;83(4):629-35.

[11] Chandler DB, Gausas RE. Lower eyelid reconstruction. Otolaryngo Clin North Am 2005;38(5):1033-42. 